\title{
An inverse approach for magnetic material characterization of an EI core electromagnetic inductor
}

\begin{tabular}{|r|l|}
\hline Journal: & Transactions on Magnetics - Conferences \\
\hline Manuscript ID: & MAGCON-09-06-0784.R1 \\
\hline Manuscript Type: & Soft Magnetic Materials $19-2009$ \\
\hline Keywords: & $\begin{array}{l}\text { Inverse problems, Magnetic measurements, Magnetic material } \\
\text { identification, Hysteresis identification }\end{array}$ \\
\hline \multicolumn{2}{|l}{} \\
\end{tabular}

\section{(5) ScholaronE \\ Manuscript Central}




\begin{abstract}
In this paper, the complete magnetic material characteristic, hysteretic and anhysteretic, is reconstructed for an EI electromagnetic inductor. The material identification process, including air gap assessment, is carried out using a coupled experimental-numerical inverse technique, based on a set of well chosen global and local magnetic measurements. It is shown that a higher accuracy is obtained when local measurements are performed in regions with less stray fields, and the air gap assessment is strongly improved by the use of local magnetic measurements.
\end{abstract}

Index Terms-Inverse problems, magnetic measurements, magnetic material identification, hysteresis identification.

\section{INTRODUCTION}

$A$ $\mathrm{N}$ EI core inductor is an electromagnetic device (EMD), which is widely used in modern power electronics applications, such as switched mode power supplies. In order to precisely design and analyze such applications, the magnetic material characteristic of the EI inductor has to be known.

Classically, the magnetic material characteristic of an EMD is determined by means of Epstein frame, single sheet tester or ring core measurements on a separate sheet of the same material as the EMD [1]. This requires extra samples of the electrical steel sheet, which are unfortunately sometimes not available. Therefore, it is convenient to characterize the magnetic properties on the specific geometry of the EMD itself. However, the non-uniform electromagnetic field patterns make this task much more difficult.

The authors propose an accurate combined numericalexperimental methodology in order to identify the magnetic properties in geometries with non-uniform electromagnetic fields. The presented methodology is applied to an EI core inductor, as shown in Fig. 1. We employ two magnetic material characteristics, each with a limited number of parameters: the anhysteretic $B-H$ curve, and the hysteresis characteristics described by the Preisach model [2].

The proposed procedure is based on a coupled experimentalnumerical inverse technique, starting from a set of global and/or local magnetic measurements and a 3D finite element model of the EI inductor, by minimizing the error between the measured and simulated electromagnetic quantity for different values of the excitation current. Depending on the nature of the measurements, (e.g. local, global, measurement position, etc.), that are used as input for the inverse problem, a certain resolution or accuracy of the recovered magnetic material

Corresponding author: A. Abdallh (E-mail: Ahmed.Abdallh@UGent.be). This work is supported by projects FWO-G.0082.06, GOA07/GOA/006, and IAP-P6/21. P. Sergeant is a postdoctoral researcher of the FWO.

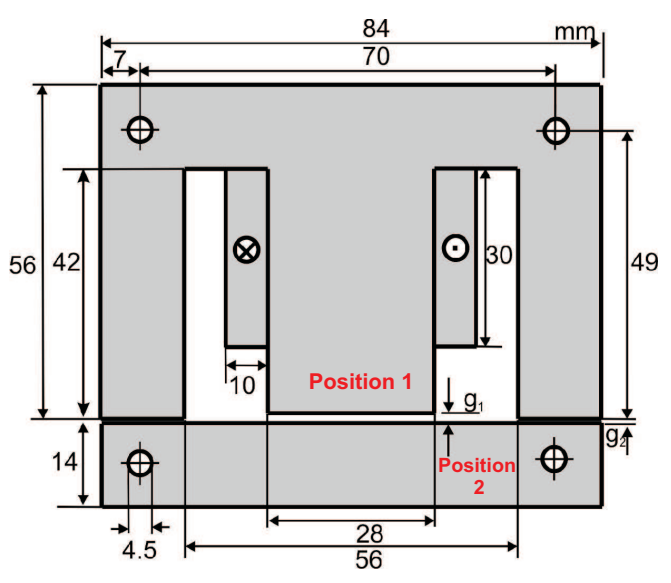

Fig. 1. The studied EI core. At positions 1 and 2 the local magnetic measurements are carried out. $g_{1}$ and $g_{2}$ are the middle and the outer air gap thicknesses, respectively. Dimensions are in $\mathrm{mm}$.

parameters is achieved. Furthermore, due to the fact that the accuracy of the magnetic material identification process is highly influenced by the uncertainty of the EMD geometry, especially the air gap thickness, we include different air gap thicknesses in the identification process. The proposed inverse approach is validated by comparing the recovered characteristics with the measured characteristics on a magnetic ring core of the same material as the EI core.

\section{PRoblem Definition}

\section{A. The studied geometry}

Fig. 1 shows the profile of the studied geometry. It is an EI core with one "middle" air gap in the middle limb $\left(g_{1}\right)$, and two "outer" air gaps $\left(g_{2}\right)$ between E and I yokes. The excitation coil is wound over the middle limb of the E-core with $N_{1}=356$ turns.

\section{B. Magnetic measurements}

The quasi static magnetic measurements are performed at $1 \mathrm{~Hz}$ for a sinusoidal current excitation, in order to have a negligible presence of eddy current effects in the magnetic core.

1) Local magnetic measurements: The local magnetic induction measurement ' $B_{\text {local }}$ ' is done by means of extra needle probes or a search coil. ' $B_{\text {local' }}$ ' is measured at different positions of the EI inductor, e.g. positions 1 and 2, as shown in Fig. 1. The positioning of the local measurement setup has a placement error of $0.01 \mathrm{~mm}$. 
2) Global magnetic measurements: The term global measurements means the measurement of two quantities; the excitation current (I) in and the voltage (V) over the excitation winding. This global measurement gives rise to the flux density linked with the excitation winding:

$$
B(t)=\frac{1}{N_{1} S} \int_{0}^{t}[V(\tau)-R I(\tau)] d \tau
$$

where $N_{1}$ is the number of turns of the excitation winding, $S$ is the cross section of the excitation winding, $R$ is the resistance of the excitation coil.

\section{Finite element modeling}

The numerical model of the EI core inductor is constructed using the 3D finite element method (FEM) which solves the nonlinear quasi-static Maxwell's equation:

$$
\nabla \times\left(\frac{1}{\mu(\mathbf{A})} \nabla \times \mathbf{A}\right)=\mathbf{J}
$$

for the magnetic vector potential $\mathbf{A}$ with the non-linear magnetic permeability $\mu$, and the current density $\mathbf{J}$. We implemented two material models, that define $\mu$ in (2).

1) Anhysteretic characteristic: The single-valued nonlinear constitutive relation of the magnetic material, is modelled by means of three parameters $\left[H_{0}, B_{0}, \nu\right]$ :

$$
\frac{H}{H_{0}}=\left(\frac{B}{B_{0}}\right)+\left(\frac{B}{B_{0}}\right)^{\nu}
$$

2) Hysteretic characteristic: The hysteretic magnetic material behavior can be fully characterized by a Preisach distribution function (PDF). Theoretically, the PDF can be identified either from an Everett map or by fitting parameters in an analytical expression. In order to limit the number of parameters to be recovered with an acceptable hysteresis model accuracy, we prefer to use an analytical expression for the PDF.

Here, the scalar Preisach model with one input (the magnetic field) and one output (the magnetic induction) is used, in which the material is constituted by several dipoles each with a non symmetric loop with up and down switching fields $\alpha$ and $\beta(\beta \leq \alpha)$. We suppose that $(\alpha, \beta)$ has a Lorentzian distribution with a reversible and an irreversible part: $P(\alpha, \beta)=P_{i r r}(\alpha, \beta)+P_{r e v}(\alpha, \beta)$.

$$
P(\alpha, \beta)=\frac{k_{1}}{\left(1+\left(\frac{\alpha-a}{b}\right)^{2}\right)\left(1+\left(\frac{\beta+a}{b}\right)^{2}\right)}+\delta_{\alpha, \beta} \frac{k_{2}}{1+\left(\frac{\alpha}{c}\right)^{2}}
$$

This PDF is characterized by a set of five Preisach parameters: $\left[a, b, c, k_{1}, k_{2}\right] . \delta_{\alpha, \beta}$ is the Kronecker delta symbol [3]. Integrating this PDF equals the magnetization saturation. These five parameters are independent as we assume that there is no a priori knowledge of the material available. Coupling the Preisach model with eq. (2) is done in a similar way as described in [4].

\section{INVERSE PROBLEM FORMULATION}

The three parameters of the anhysteretic $B$ - $H$ characteristic $\boldsymbol{p}=\left[H_{0}, B_{0}, \nu\right]$, and the five parameters of the Lorentzian distribution of the scalar Preisach model $\boldsymbol{p}=\left[a, b, c, k_{1}, k_{2}\right]$ are recovered using the proposed inverse approach. In the following section, three objective functions $O F$ are formulated, which minimize iteratively the quadratic error between the measured and simulated quantities:

$$
\boldsymbol{p}^{\star}=\arg \min _{\boldsymbol{p}} O F(\boldsymbol{p})
$$

where $\boldsymbol{p}^{\star}$ is the optimum 'recovered' material parameters.

\section{A. Objective Functions}

The first and second objective function $\left(O F_{1}, O F_{2}\right)$ are implemented using the amplitude $\left(i_{k}\right)$ of the $k^{\text {th }}$ sinusoidal excitation current $(k=1, . ., K=40)$ and the local magnetic induction measurements, at fixed positions, e.g. position 1 or position 2 of Fig. 1, respectively:

$$
\begin{aligned}
& O F_{1}(\boldsymbol{p})=\sum_{k=1}^{K}\left\|\frac{B_{s}\left(i_{k}, \boldsymbol{p}\right)-B_{m}\left(i_{k}\right)}{B_{m}\left(i_{k}\right)}\right\|_{\text {position } 1}^{2} \\
& O F_{2}(\boldsymbol{p})=\sum_{k=1}^{K}\left\|\frac{B_{s}\left(i_{k}, \boldsymbol{p}\right)-B_{m}\left(i_{k}\right)}{B_{m}\left(i_{k}\right)}\right\|_{\text {position } 2}^{2}
\end{aligned}
$$

with $B_{m}\left(i_{k}\right)$ the measured peak magnetic induction value of the $k^{t h}$ excitation current and $B_{s}\left(i_{k}, \boldsymbol{p}\right)$ the corresponding simulated local flux densities using the 3D FEM and the material parameter values $\boldsymbol{p}$.

The third objective function $\left(\mathrm{OF}_{3}\right)$ is implemented using global measurements of the excitation current $(I)$ and the voltage $(V)$ of the excitation winding, see eq. (1), where no local measurements are used:

$$
\begin{gathered}
\phi_{m}(t)=\left(1 / N_{1}\right)\left[\int_{0}^{t} V(\tau) d \tau-R \int_{0}^{t} I(\tau) d \tau\right] \\
O F_{3}(\boldsymbol{p})=\sum_{k=1}^{K}\left\|\frac{\phi_{s}\left(i_{k}, \boldsymbol{p}\right)-\phi_{m}\left(i_{k}\right)}{\phi_{m}\left(i_{k}\right)}\right\|^{2}
\end{gathered}
$$

with $\phi_{m}\left(i_{k}\right)$ the measured peak magnetic flux value of the $k^{\text {th }}$ excitation current and $\phi_{s}\left(i_{k}, \boldsymbol{p}\right)$ the corresponding simulated value.

\section{B. Combined objective functions}

To increase the efficiency of the numerical inverse approach, we combine the previous objective functions, so that more information is given as input to the inverse approach. Each objective function is based on two measured quantities only, and the excitation current is the common factor in all of them: $\left(I, B_{\text {local }-1}\right),\left(I, B_{\text {local-2 }}\right)$, and $(I, V)$. In the combined objective function $(C O F)$, we combine two or three previously mentioned objective functions:

$$
\begin{gathered}
C O F_{12}=O F_{1} \cup O F_{2} \\
C O F_{13}=O F_{1} \cup O F_{3} \\
C O F_{23}=O F_{2} \cup O F_{3} \\
C O F_{123}=O F_{1} \cup O F_{2} \cup O F_{3}
\end{gathered}
$$


Fig. 2. The anhysteretic $B-H$ characteristic using $O F_{1}, O F_{2}$, and $O F_{3}$ compared to the reference characteristic, $\left(g_{1}=0.85 \mathrm{~mm}, g_{2}=0\right)$.

\section{RESULTS AND DisCUSSION}

The results of the magnetic material identification based on the proposed inverse approach are presented and discussed in the following sections.

\section{A. Magnetic material identification (Anhysteretic behavior)}

In this part, we solve the inverse problem in order to identify the three parameters $\left[H_{0}, B_{0}, \nu\right]$. Here, the value of the middle and outer air gap thicknesses are kept constant $\left(g_{1}=0.85 \mathrm{~mm}, g_{2}=0\right)$. Values are given by the constructor of the EI profile.

Fig. 2 shows the reconstructed anhysteretic $B-H$ characteristic of the EI core inductor material using the proposed inverse method by implementing $O F_{1}, O F_{2}$, and $O F_{3}$.

The recovered characteristic is compared to the measured 'reference' magnetic parameters using the ring core measurements of the same material as the EI core material.

A good correspondence between the recovered magnetic parameters and the reference magnetic parameters, particularly at saturation, is observed. The best results are observed for $O F_{2}$, which is based on the local magnetic induction at the Icore. $O F_{1}$, which is based on the local magnetic induction at the middle limb, and $O F_{3}$, which is based on global measurements slightly differ from the reference characteristic.

These results can be explained as follows: the inverse problem is generally an ill-posed problem, i.e. a small error in the measured quantities leads to an error in the recovered parameters. $O F_{1}$ is based on $B_{\text {local-1 }}$ near to the middle air gap. It can be affected by the fringing effect. $O F_{3}$ is based on $V$ over the excitation winding. It can be affected by the value of the ohmic resistance $R$ in (1). However, $O F_{2}$ is based on $B_{\text {local }-2}$ on the I-core. It is far from the excitation winding, and hence it is hardly affected by the stray fields. Therefore, solving the inverse problem based on the most reliable measurements gives the best results.

\section{B. Magnetic material identification (Anhysteretic behavior) and one air gap assessment}

In this part, we solve the inverse problem in order to identify the three parameters $\left[H_{0}, B_{0}, \nu\right]$. Moreover, the difficulty of the inverse problem is increased by including the value of the middle air gap $\left(g_{1}\right)$ as unknown in the identification process. The value of the outer air gap thickness is kept constant $\left(g_{2}=\right.$ $0)$.

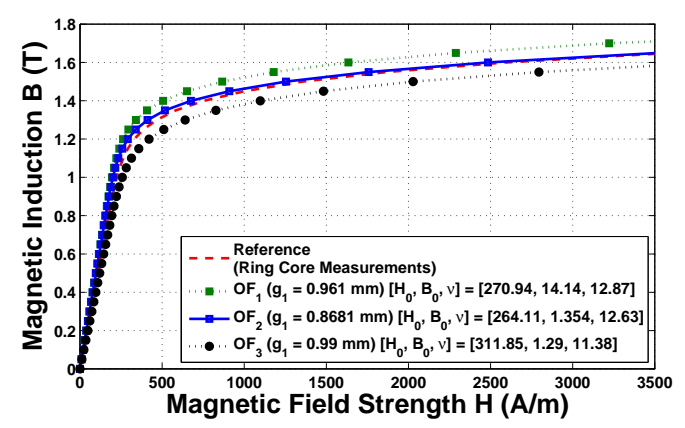

Fig. 3. The anhysteretic $B-H$ characteristic using $O F_{1}, O F_{2}$, and $O F_{3}$ compared to the reference characteristic, $\left(g_{1}\right.$ is unknown, $\left.g_{2}=0\right)$.

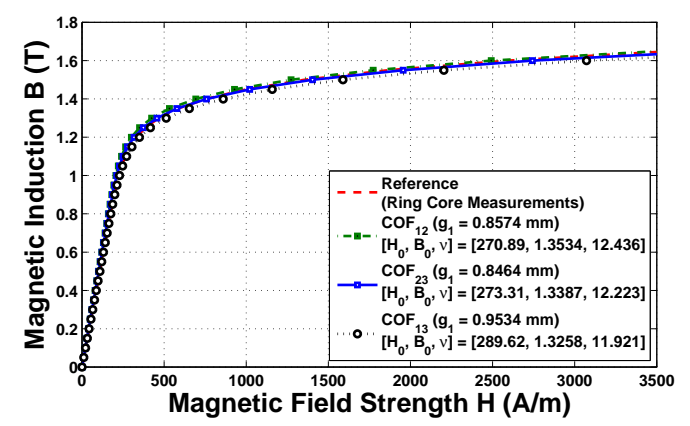

Fig. 4. The anhysteretic $B-H$ characteristic using $C O F_{12}, C O F_{13}$, and $C O F_{23}$ compared to the reference characteristic, $\left(g_{1}\right.$ is unknown, $\left.g_{2}=0\right)$.

Fig. 3 shows the reconstructed anhysteretic $B-H$ characteristic of the EI core inductor material using the proposed inverse method by implementing $O F_{1}, O F_{2}$, and $O F_{3}$, compared to the 'reference' magnetic parameters.

It can be observed from Fig. 3 that solving the inverse problem based on these objective functions does not guarantee the accurate magnetic parameters and the accurate middle air gap thickness value.

In order to well pose the inverse problem, we need to include more measurements as input of the inverse problem, i.e. use of the combined objective functions $C O F_{12}, C O F_{13}$, and $C O F_{23}$, see equ. (10-12). Fig. 4 depicts the reconstructed anhysteretic $B-H$ characteristic of the EI core inductor material using the proposed inverse method by implementing $C O F_{12}$, $C O F_{13}$, and $C O F_{23}$, compared to the 'reference' magnetic parameters.

It can be observed from Fig. 4 that $C O F_{12}$, and $C O F_{23}$ result in accurate material parameters, as well as the accurate middle air gap thickness. However, $\mathrm{COF}_{13}$ results in accurate material parameters, and inaccurate middle air gap thickness. At position 2, very accurate measurements are provided to the inverse problem, see also sec. IV-A.

\section{Magnetic material identification (Anhysteretic behavior) and two air gaps assessment}

In this part, we solve the inverse problem in order to identify the three parameters $\left[H_{0}, B_{0}, \nu\right]$, and the two unknown air gaps thicknesses ( $g_{1}$ and $g_{2}$ are unknown). The magnetic measurements were carried out for two measured values of the outer air gap thickness, i.e. $g_{2}=0.25 \mathrm{~mm}$, and $g_{2}=0.5 \mathrm{~mm}$, 


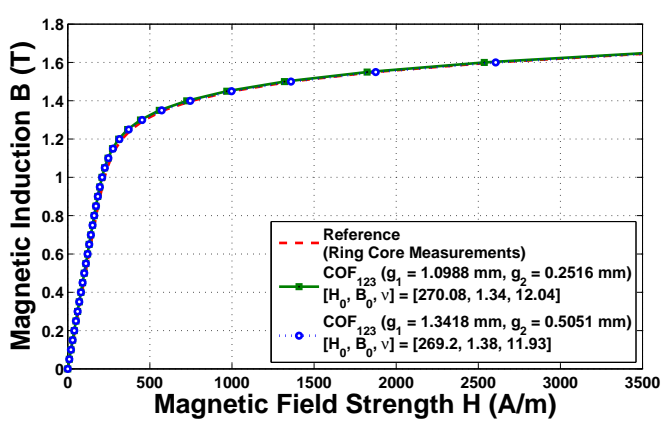

Fig. 5. The anhysteretic $B-H$ characteristic using $C O F_{123}$ compared to the reference characteristic, ( $g_{1}$ and $g_{2}$ are unknown).

by putting plastic spacers with known thickness between the $\mathrm{E}$ and I yokes. The two yokes are fixed together by a mechanical clamp to prevent the movement of the I-yoke.

Fig. 5 shows the reconstructed anhysteretic $B-H$ characteristic of the EI core inductor material using the proposed inverse method, by implementing $C O F_{123}$, compared to the 'reference' magnetic parameters. It can be observed from Fig. 5 that $C O F_{123}$ results in accurate material parameter identification, as well as the accurate air gap thickness for both examined outer air gap thicknesses, i.e. $g_{2}=0.25$, and $0.5 \mathrm{~mm}$.

\section{Magnetic material identification (Hysteretic behavior)}

In this part, we solve the inverse problem in order to identify the five parameters of the Lorentzian distribution of the scalar Preisach model $\left[a, b, c, k_{1}, k_{2}\right]$. The value of the middle and outer air gap thicknesses are kept constant $\left(g_{1}=0.85 \mathrm{~mm}, g_{2}=0\right)$, respectively. For simplicity, we have tested only one objective function, i.e. $O F_{2}$, which provides the most accurate measurements.

$$
O F_{2}(\boldsymbol{p})=\sum_{l=1}^{L} \sum_{n=1}^{T}\left\|\frac{b_{s, l}\left(t_{n}, \boldsymbol{p}\right)-b_{m, l}\left(t_{n}\right)}{b_{m, l}\left(t_{n}\right)}\right\|_{\text {position } 2}^{2}
$$

where $b_{m, l}\left(t_{n}\right)$ is the measured magnetic induction value of the $n^{\text {th }}$ time step $(n=1, \ldots, T=1000)$ for the $l^{\text {th }}$ magnetization loop and $b_{s, l}\left(t_{n}, \boldsymbol{p}\right)$ is the corresponding simulated local flux densities using the 3D FEM.

Fig. 6 shows the recovered hysteresis loops of the EI core inductor material using the proposed inverse method, by implementing $O F_{2}$, compared to the 'reference' magnetic hysteresis loops. A good correspondence between the measured hysteresis loops on the ring core, and the recovered one is observed. Table I indicates the five Lorentzian parameters fitted on the magnetic ring core measurements data, and the corresponding parameters recovered from the inverse problem.

From table I, it is clear that the presented method to identify the parameters of the Preisach model results in a deviation less than $10 \%$ between the identified and the reference parameter values. In [5], the inverse problem is solved for a rather simple geometry, i.e. no air gap. Moreover, it has been assumed, in [5], that the hysteresis (magnetic field - magnetic induction) loops have a direct relation with the measured current-flux loops according to Ampere's and Faraday's laws, respectively. However, this assumption is not valid for an EMD with highly

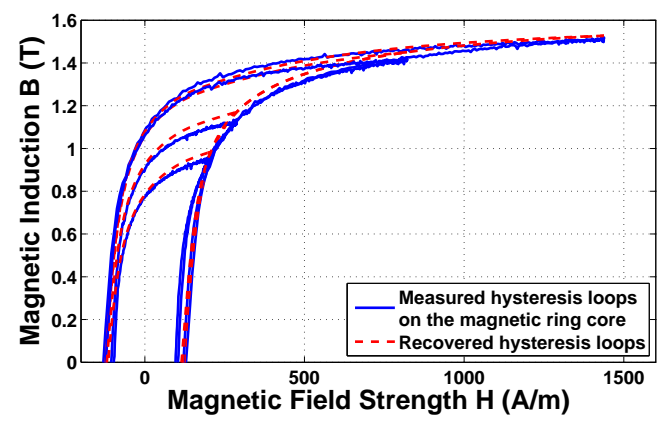

Fig. 6. The hysteresis loops using $\mathrm{OF}_{2}$ compared to the reference characteristic, $\left(g_{1}=0.85 \mathrm{~mm}, g_{2}=0\right)$.

non-uniform field patterns. On the other hand, our proposed method is general and can be applied to any EMD.

TABLE I

THE LORENTZIAN PARAMETERS OF THE PREISACH MODEL COMPARED TO THE FITTED PARAMETERS ON THE MEASUREMENTS DATA

\begin{tabular}{cccccc}
\hline \hline Parameter & $a$ & $b$ & $c$ & $k_{1} \times 10^{-4}$ & $k_{2} \times 10^{-4}$ \\
\hline Fitted value & 124.99 & 38.01 & 504.08 & 1.89 & 5.15 \\
\hline Recovered value & 123.32 & 39.01 & 480 & 1.8 & 5.52 \\
\hline \hline
\end{tabular}

\section{CONCLUSION}

In this paper, we proposed a method to reconstruct the hysteretic and the anhysteretic behavior of an EMD. An EI core inductor was used as a test case. The proposed method solves an inverse problem starting from well defined global and/or local magnetic measurements, followed by a numerical procedure, that is based on the finite element method. Moreover, assessment of the unknown air gaps is investigated and validated. The identification of the hysteretic and the anhysteretic behavior gives a good indication for the hysteresis losses and the magnetic permeability, respectively. The numerical inverse problem approach has been found to be an effective methodology for the extraction of the magnetic characteristics of the magnetic material.

\section{REFERENCES}

[1] E. Antonelli, E. Cardelli, and A. Faba, "Epstein frame: How and when it can be really representative about the magnetic behavior of laminated magnetic steels," IEEE Trans. on Magnetics, Vol. 41, No. 5, pp. 15161519, May 2005.

[2] I. D. Mayergoyz, Mathematical Models of Hysteresis. Berlin: Springer, 1991.

[3] B. Azzerboni, E. Cardelli, G. Finocchio, and F. La Foresta, "Remarks about preisach function approximation using lorentzian function and its identification for nonoriented steels," IEEE Trans. on Magnetics, Vol. 39, No. 5, pp. 3028-3030, September 2003.

[4] P. Sergeant, and L. Dupré, "Implementation of hysteresis material characteristics in finite element computations," Proceeding COMSOL Users conference, Grenoble, 2007. Available online http://cds.comsol.com/access/dl/papers/2604/Sergeant.pdf

[5] M. Toman, G. Štumberger, and D. Dolinar, "Parameter identification of the Jiles-Atherton hysteresis model using differential evolution," IEEE Trans. on Magnetics, Vol. 44, No. 6, pp. 1098-1101, June 2008. 


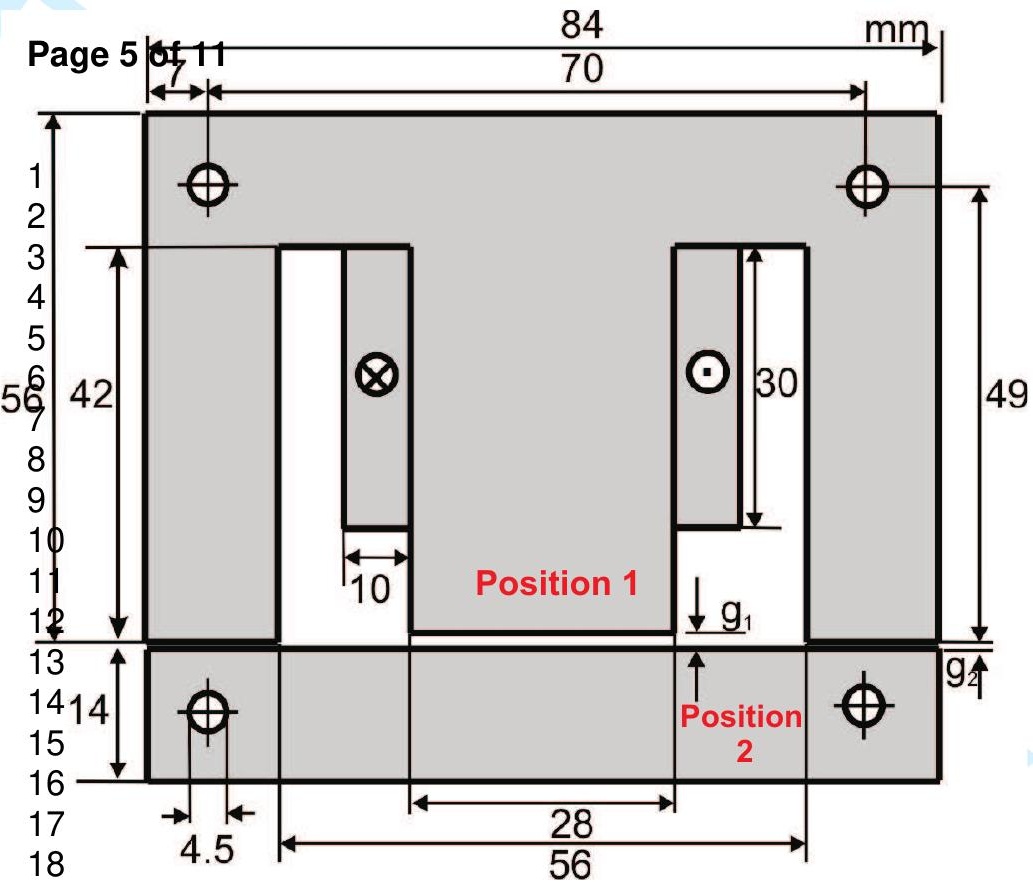




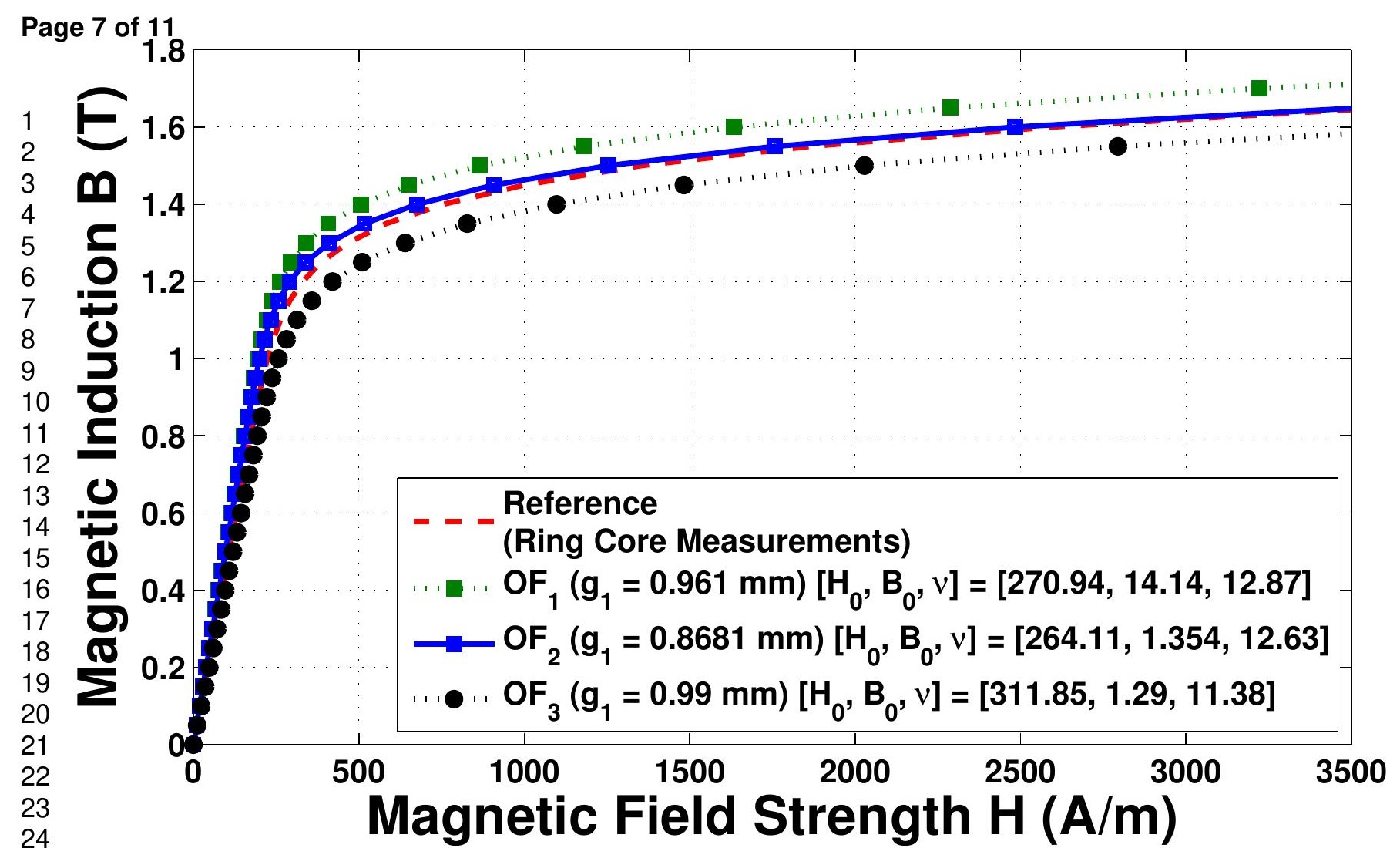




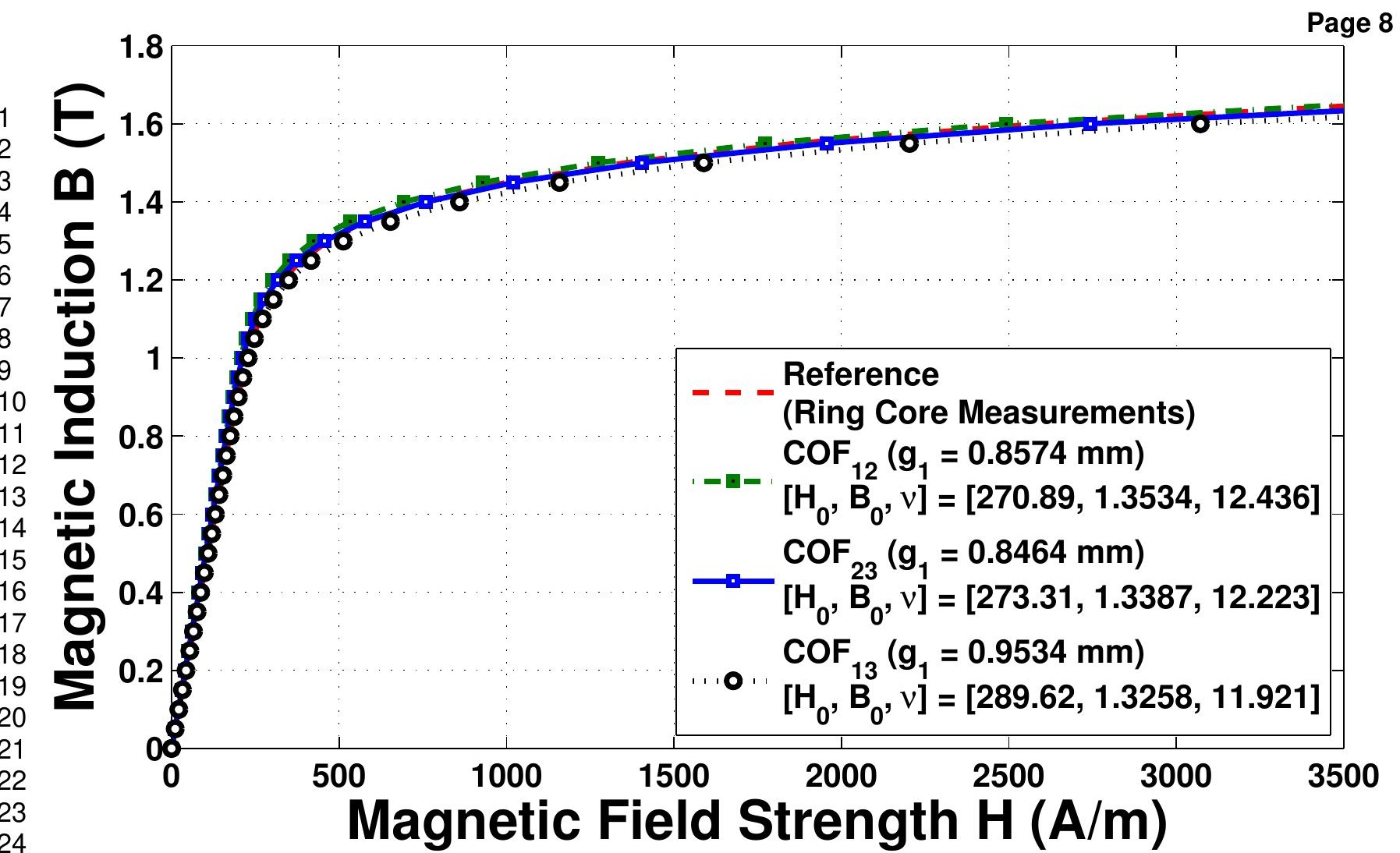




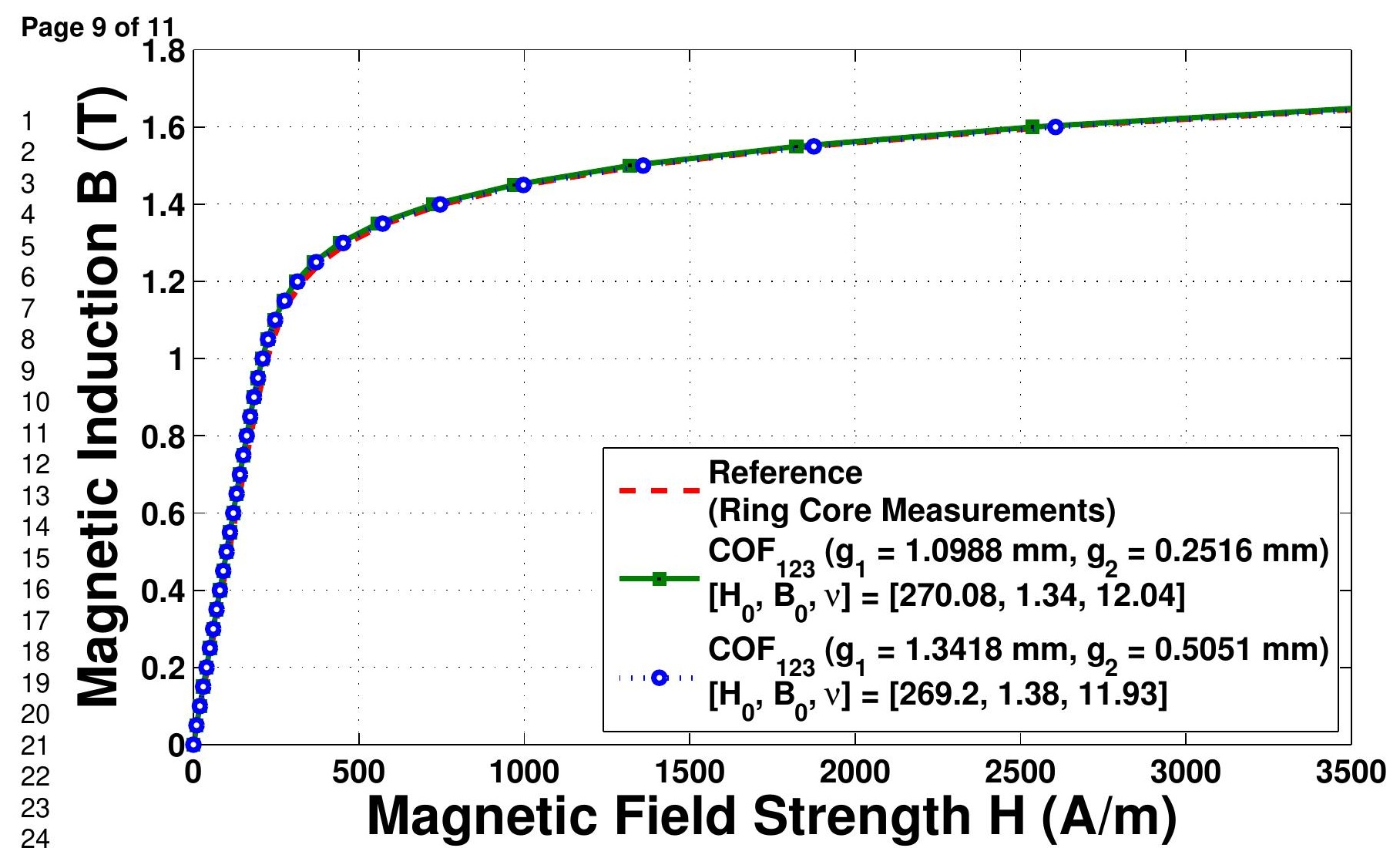




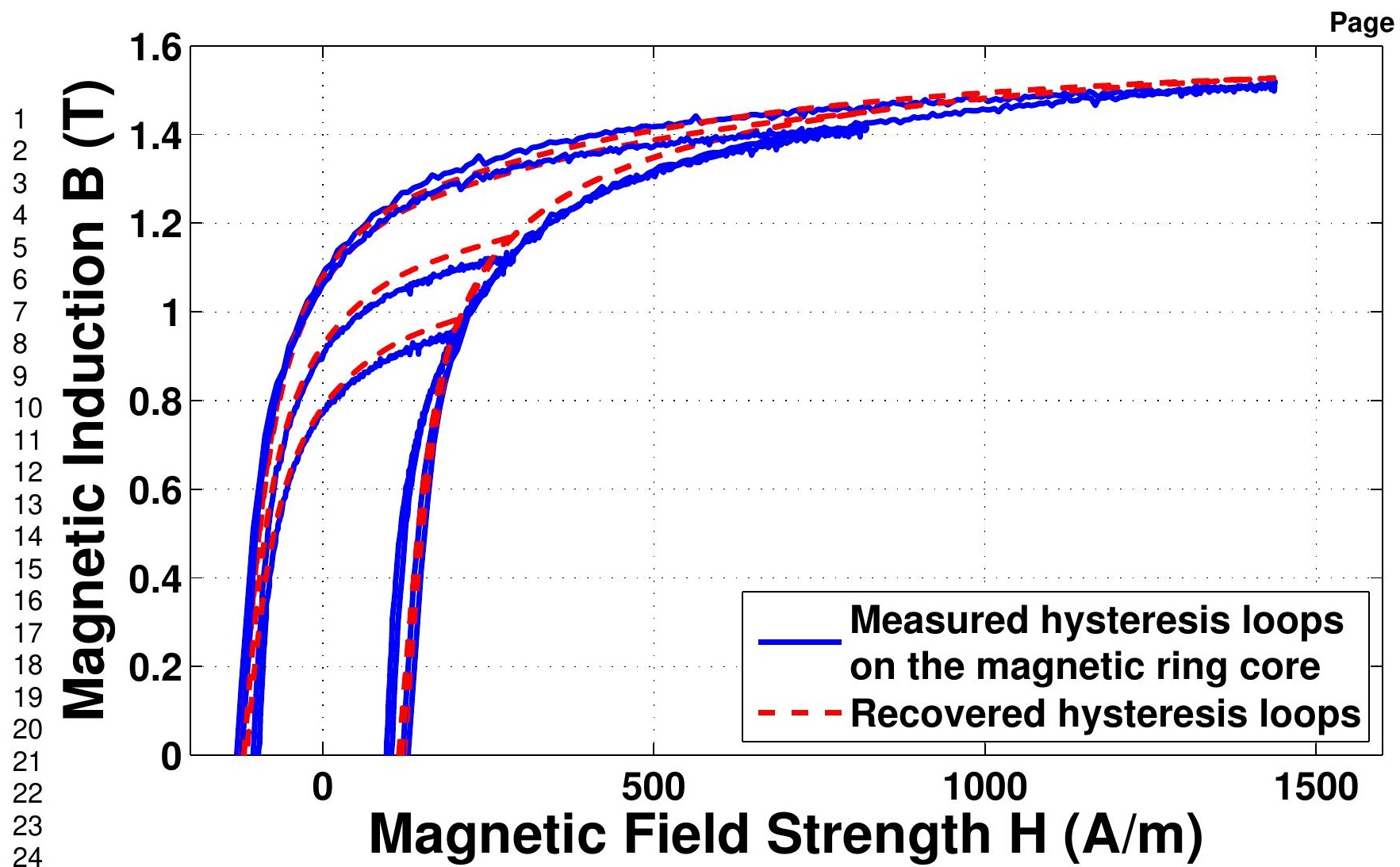




\section{Authors Contact Information Sheet}

\begin{tabular}{|l|l|l|l|}
\hline Order & \multicolumn{1}{|c|}{ Name } & \multicolumn{1}{c|}{ E-mail } & \multicolumn{1}{c|}{ Phone number } \\
\hline 1 & Ahmed Abou-Elyazied Abdallh & Ahmed.Abdallh@ugent.be & $+32(0) 92643420$ \\
\hline 2 & Peter Sergeant & Peter.Sergeant@ugent.be & $+32(0) 92643424$ \\
\hline 3 & Guillaume Crevecoeur & Guillaume.Crevecoeur@ugent.be & $+32(0) 92643421$ \\
\hline 5 & Luc Dupré & Luc.Dupre@ugent.be & $+32(0) 92643424$ \\
\hline
\end{tabular}

Affiliation of all authors:

Ghent University, Department Electrical Energy, Systems and Automation (EESA)

Electrical Energy Laboratory (EELAB)

Sint-Pietersnieuwstraat 41, B-9000 Gent, Belgium.

Fax Number of all authors:

+32(0)9 2643582

Corresponding author:

Ahmed Abou-Elyazied Abdallh 